$\mathrm{E}$

EVALUAR
2018, Vol. 18, No. 2

ISSN 1667-4545

Recuperado de https://revistas.unc.edu.ar/index.php/revaluar

Laboratorio de Evaluación Psicológica y Educativa

Facultad de Psicología - Universidad Nacional de Córdoba

\title{
Desarrollo y validación de un instrumento para medir discriminación percibida en la comunidad LGBT
}

\section{Development and Validation of an Instrument for the Measurement of Perceived Discrimination in the LGBT Community}

\author{
Juan Aníbal González-Rivera * 1, Suham Pabellón-Lebrón ${ }^{2}$ \\ 1- Ponce Health Sciences University, Ponce, Puerto Rico \\ 2- Universidad Interamericana de Puerto Rico, Recinto Metropolitano, Puerto Rico.
}

Introducción

Marco conceptual

Resultados

Discusión

Referencias

Recibido: 24/03/2018 Revisado: 10/05/2018 Aceptado: 28/05/2018

\section{Resumen}

El presente estudio analiza las propiedades psicométricas de la Escala de Discriminación Percibida en la Comunidad LGBT (Lesbianas, Gays, Bisexuales y Transgénero) en una muestra de adultos puertorriqueños. Un total de 305 miembros de la comunidad LGBT participaron en este estudio de carácter exploratorio y psicométrico. Los resultados confirmaron que la escala posee una estructura de dos factores. Estos factores son discriminación individual y discriminación grupal. Un total de 20 ítems cumplieron con los criterios de discriminación y cargas factoriales apropiadas (10 ítems por factor). El índice de confiabilidad alfa de Cronbach para ambas subescalas fue .89. Estos resultados sugieren que la escala tiene el potencial para medir este constructo en miembros de la comunidad LGBT en Puerto Rico.

Palabras clave: discriminación, LGBT, homosexualidad, propiedades psicométricas, validación

\begin{abstract}
This article examines the psychometric properties of the Discrimination Perceived in the LGBT (Lesbians, Gays, Bisexuals and Transgender) Community Scale in a sample of Puerto Rican adults. A total of 305 individuals from the LGBT community participated in this exploratory and psychometric study. The results indicated that the scale fits better into a model of two factors. These factors are Personal Discrimination and Group Discrimination. A total of 20 items matched the criteria of discrimination and presented appropriate factor loadings (10 items by factor). The reliability index of the subscales was .89 (Cronbach's alpha). These results suggest that the instrument has the potential to measure this construct among members of the LGBT community in Puerto Rico.
\end{abstract}

Keywords: discrimination, LGBT, homosexuality, psychometric properties, validation

\footnotetext{
*Correspondencia a: Dr. Juan A. González-Rivera. Dirección Postal: 500 West Main Suite 215, Bayamón, Puerto Rico, 00961. Tel.: 011787315 6034. e-mail: dr.juananibalgonzalez@outlook.com

Cómo citar este artículo: González-Rivera, J. A., \& Pabellón-Lebrón, S. (2018). Desarrollo y validación de un instrumento para medir discriminación percibida en la comunidad LGBT. Revista Evaluar, 18(2), 59-74. Recuperado de https://revistas.unc.edu.ar/index.php/revaluar

Nota de Autor:

Los autores expresan que no hubo conflictos de intereses al redactar el manuscrito. Para permiso de uso y descargar el instrumento, visite www.juananibalgonzalez.com.
} 


\section{Introducción}

En Puerto Rico, así como en muchos países del mundo, la comunidad LGBT (lesbianas, gays, bisexuales, transgénero y demás orientaciones sexuales distintas de la heterosexual) es víctima de discriminación, prejuicio y rechazo social. Incluso, a pesar de los múltiples eventos históricos protagonizados por miembros de la comunidad LGBT en búsqueda de equidad, la heterosexualidad y la identidad de género relacionada con el sexo biológico continúan siendo vistos como las formas sociales y culturales normalmente establecidas (Francia-Martínez, Esteban, \& Lespier, 2017). Dentro de la sociedad puertorriqueña, este fenómeno, también llamado "heteronormalidad", es causante de la valoración negativa, convertida en prejuicio, en referencia a la orientación sexual del individuo homosexual, lesbiana o bisexual (Fernández-Rodríguez \& Calderón-Squiabro, 2014).

La literatura científica confirma que la discriminación, tanto en contra de la comunidad LGBT como de otros grupos marginados, es en la actualidad un fenómeno social ampliamente extendido que tiene terribles consecuencias en la salud mental de las personas discriminadas (Burges, Lee, Tran, \& Van Ryn, 2007; Sandfort, de Graaf, \& Bij1, 2003; Singh \& Dickey, 2016; Woodford, Paceley, Kulick, \& Hung, 2015). A pesar de que estas nocivas consecuencias para el bienestar psicológico son conocidas (Bostwick, Boyd, Hughes, West, \& McCabe, 2014; Fingerhut, Peplau, $\&$ Gable, 2010), en Puerto Rico no existe una escala diseñada para medir la discriminación percibida por los miembros de la comunidad LGBT. El desarrollo y validación de una escala de este tipo ayudaría a la comunidad científica puertorriqueña a conocer el sentir de este grupo para brindar apoyo en la creación de posibles organizaciones, de programas institucionales y de políticas públicas a favor de la comunidad LGBT.

Teniendo esto en consideración, este proyecto tuvo como objetivo principal desarrollar y validar un instrumento que mida, específicamente, discriminación percibida individual (experiencias personales de discriminación) y discriminación percibida grupal (acciones discriminatorias hacia el colectivo LGBT). A estos efectos, el presente estudio se orienta a comprobar si las propiedades psicométricas del instrumento avalan su utilización en contextos clínicos e investigativos. También se intenta determinar si la distribución factorial del instrumento evidencia ambas dimensiones propuestas, discriminación individual y discriminación grupal. Con este fin, se analizaron las propiedades psicométricas del instrumento. Esto permitirá ofrecer a la comunidad científica un instrumento válido y confiable que contribuya al avance del estudio de este fenómeno en Puerto Rico y América Latina.

\section{Marco Conceptual}

La discriminación percibida se define como la experiencia subjetiva y cognitiva de sentirse víctima de discriminación en un contexto sociocultural e histórico que se actualiza mediante el discurso (González-Rivera, Pabellón-Lebrón, \& Rosario-Rodríguez, 2017). Por otra parte, el acrónimo LGBT se utiliza para denominar varias orientaciones sexuales e identidades de género, y cada una de sus letras se refiere a una población diferente (lesbianas, gays, bisexuales y transgénero). Existen diversas variaciones de este acrónimo, entre ellas LGBTT, para incluir a la población transexual, LGBTTQ, que hace referencia al género "queer", LGBTTQQ, para incluir a las personas autodenominadas como "questioning", y también puede encontrarse el acrónimo LGBTTQQI que incluye entre sus siglas a la población 
intersexual. Para el propósito de esta investigación, se utilizará el acrónimo LGBT en referencia todas las poblaciones anteriormente mencionadas.

La discriminación percibida por la comunidad LGBT se define como la experiencia subjetiva y cognitiva de personas homosexuales, lesbianas, bisexuales, transgénero, transexuales, "queer", "questioning" e intersexuales de sentirse víctimas de discriminación en un contexto sociocultural e histórico que se actualiza mediante el discurso. La mayoría de las investigaciones sobre discriminación subdividen este constructo en dos dimensiones: discriminación individual y discriminación grupal (Armenta \& Hunt, 2009; González-Rivera et al., 2017). La primera se refiere a las experiencias personales de discriminación, entiéndase conductas discriminatorias dirigidas particularmente hacia un individuo, mientras que la segunda se relaciona con las acciones y conductas discriminatorias dirigidas hacia el colectivo en su conjunto (p. ej., manifestaciones masivas, políticas públicas, leyes y otorgamiento o eliminación de derechos en una sociedad).

\section{Discriminación hacia la Comunidad LGBT}

Una de las principales razones por la que ha sido difícil proteger los derechos de la comunidad LGBT en Puerto Rico es el fuerte cabildeo de sectores religiosos, políticos tradicionales y fundamentalistas, y las políticas públicas que generalmente no promueven la equidad (Rodríguez-Madera, Ramos-Pibernus, Padilla, \& Varas-Díaz, 2016). Más allá de meras suposiciones antagónicas, se ha encontrado que las creencias religiosas fundamentalistas y tradicionales están directamente relacionadas con altos niveles de estigma y rechazo hacia la comunidad LGBT (Nazario-Serrano, 2016), mientras que la identificación con un grupo religioso y la participación de actividades religiosas están asociadas a la manifestación de actitudes homofóbicas y a mayor distancia social hacia esta comunidad (Fernández-Rodríguez \& Calderón-Squiabro, 2014; Nieves-Rosa, 2012).

Peyorativos como "loca", "pato" y "maricón" son comúnmente utilizados para devaluar a estos individuos por su orientación sexual (González-Guardarrama \& Toro-Alfonso, 2012). Según participantes de un estudio realizado por Luiggi-Hernández et al. (2015), las experiencias ofensivas frecuentemente reportadas incluyeron burlas $(48 \%)$, comentarios negativos sobre la comunidad LGBT (37\%), preguntas constantes sobre su orientación sexual (34\%) y hostigamiento sexual (21\%). Este tipo de agresiones, a consecuencia de la exposición a un clima experiencial heterosexista, se asocia a mayores niveles de estrés percibido y un aumento en los síntomas relacionados con la ansiedad (Woodford et al., 2015). Conjuntamente, los individuos expuestos podrían presentar limitaciones en su lugar de trabajo y en algunos casos optar por el suicidio (González-Guardarrama \& Toro-Alfonso, 2012). De igual forma, la discriminación hacia las personas identificadas como transexuales y transgénero está ampliamente extendida en la sociedad actual y es manifestada comúnmente a través de burlas, violencia y microagresiones, que ponen a estas personas en riesgo de sufrir ansiedad, depresión, traumas, abuso de sustancias, VIH/SIDA y cometer suicidio (Singh \& Dickey, 2016).

Esta discriminación percibida por los miembros de la comunidad LGBT juega un papel determinante en la elección de hacer pública su orientación sexual, o esconderla y disfrutar de su sexualidad. Esta situación se refleja en los resultados de un estudio realizado por Rodríguez-Díaz, Jovet-Toledo, et al. (2016) en el que el 49\% de la muestra identificó el rechazo familiar como la causa principal del ocultamiento de su iden- 
tidad de género u orientación sexual. El miedo a la discriminación (41\%) y al bullying (38\%) también influyeron en las decisiones de estos individuos. En otro estudio similar realizado por González-Guardarrama y Toro-Alfonso (2012), los participantes expresaron sentir miedo por lo que dirían otras personas con respecto a sus preferencias, lo que reflejó impotencia, frustración y tristeza, y puso de relieve la importancia de las construcciones sociales y familiares. Estos participantes también mencionaron haber sido víctimas de humillaciones, marginación, prejuicios y atropellos por ser diferentes, como así también haber sentido que carecían de los mismos derechos que tienen los heterosexuales en la sociedad.

Este tipo de discriminación puede manifestarse en diversos escenarios sociales sin importar las normas generales, códigos de conducta o políticas institucionales de los mismos. Los autores Rivera-Quiñones, Toro-Alfonso y Meléndez (2013) realizaron un estudio utilizando una muestra compuesta por 103 miembros de la comunidad LGBT de los cuales 65 participantes admitieron haber evitado hacer alguna muestra de cariño o amistad a un compañero de trabajo o de clases por temor a que pudiera pensar que lo hacían por su orientación sexual, mientras que 63 participantes informaron haberse sentido obligados a cambiar el género o utilizar términos generales para referirse a sus parejas al hablar de estas con compañeros de trabajo o de clases. Por otro lado, 64 participantes mencionaron haber sido molestados por algún compañero de trabajo o de clases a causa de su orientación sexual, así como 58 de los 103 participantes mencionaron que alguna vez habían sido verbalmente insultados por esta misma razón. De los mismos resultados se desprende que 56 participantes reconocieron haber sentido miedo de hablar y participar en una conversación con desconocidos por temor a que el grupo pensara que era gay/lesbiana/transgénero.
Otros autores como Rodríguez-Díaz, Martínez-Vélez, et al. (2016) encontraron que el 50\% de los participantes de su estudio reportaron haber sido discriminados por su orientación o identidad sexual en la escuela, el 26\% de estos reportó haber percibido discriminación en sus trabajos, mientras que un 19\% indicó haber percibido discriminación al momento de solicitar servicios gubernamentales. Incluso, en el ambiente laboral, resultados como los encontrados por Rosario-Hernández, Rovira-Millán, Luna-Cruz, Neris y Acevedo (2009), exponen que, para evitar ser víctimas de discriminación por su orientación sexual, algunos empleados identificados con la comunidad LGBT utilizan estrategias de evitación y fingimiento, a pesar de que estas estén asociadas a la presencia de sintomatología depresiva y a una menor satisfacción laboral.

Por otra parte, la protección ante la discriminación por orientación sexual e identidad de género en el escenario laboral, el derecho al matrimonio entre parejas del mismo sexo y a la adopción y la protección contra la violencia de género, fueron consideradas por los miembros de la comunidad LGBT como las medidas más importantes en lo relacionado a su bienestar social (Rodríguez-Díaz, Jovet-Toledo, et al., 2016; Rodríguez-Díaz, Martínez-Vélez, et al., 2016). Cabe mencionar que el grado de aceptación social está asociado con el bienestar psicológico de los miembros de esta comunidad, mientras que su identidad de género desempeña un rol influyente en la relación entre el grado de aceptación y el bienestar psicológico (Woodford et al., 2015). Para esta comunidad, el apoyo social proporcionado por la familia y los amigos constituye un importante factor de bienestar, y se observa que, a mayor percepción de apoyo por parte de estos, menores los niveles de depresión y ansiedad y mejor autoestima (Marchueta-Perez, 2014). 
Método

Diseño

Esta investigación tiene un diseño de estudio instrumental (Ato, López, \& Benavente, 2013; Montero \& León, 2007). En ella se analizaron las propiedades psicométricas de la Escala de Discriminación Percibida en la Comunidad LGBT para su validación en Puerto Rico.

\section{Participantes}

Se trabajó con una muestra no probabilística constituida por 305 participantes de la comunidad LGBT en Puerto Rico, con una edad promedio de $34.75(\mathrm{DE}=10.578)$. En la Tabla 1 se presentan los datos sociodemográficos de los participantes. Para participar en este estudio, se establecieron los siguientes criterios de inclusión: (1) ser mayor de 21 años de edad, (2) ser residente en Puerto Rico y (3) auto-identificarse como parte de la comunidad LGBT.

\section{Instrumentos}

Cuestionario de Datos Generales. Para identificar las características sociodemográficas de la muestra, se desarrolló un cuestionario de datos generales, que recogía información importante en cuanto a la edad, sexo, orientación sexual y preparación académica.

Escala de Discriminación Percibida en la Comunidad LGBT. Este instrumento fue desarrollado por los investigadores para medir la discriminación percibida por las personas auto-identificadas como parte de la comunidad LGBT. La versión original del instrumento estaba constituida por 50 premisas distribuidas de acuerdo a un modelo de dos factores: discriminación personal y discrimi-
Tabla 1

Información sociodemográfica de la muestra.

\begin{tabular}{lrr}
\multicolumn{1}{c}{ Variables } & $\boldsymbol{f}$ & $\boldsymbol{\%}$ \\
\hline Sexo & & \\
Femenino & 94 & 30.8 \\
Masculino & 205 & 67.2 \\
Transgénero & 6 & 2.0 \\
& & \\
Preparación Académica & & \\
Escuela superior o menos & 12 & 3.9 \\
Grado asociado / técnico & 45 & 14.8 \\
Bachillerato & 126 & 41.3 \\
Maestría & 94 & 30.8 \\
Doctorado o grado equivalente & 28 & 9.2 \\
& & \\
Estado sentimental & & \\
Soltero/a & 141 & 46.2 \\
Casado/a & 33 & 10.8 \\
Noviazgo & 53 & 17.4 \\
Convivencia & 78 & 25.6 \\
Orientación Sexual & & \\
Gay & & \\
Lesbiana & 190 & 62.3 \\
Bisexual & 65 & 21.3 \\
Otra & 45 & 14.8 \\
\hline & 5 & 1.6 \\
\hline
\end{tabular}

Nota. $\mathrm{N}=305$.

nación grupal. El instrumento tiene una escala de respuesta tipo Likert de seis puntos: 1 (Totalmente en desacuerdo), 2 (En desacuerdo), 3 (Parcialmente en desacuerdo), 4 (Parcialmente de acuerdo), 5 (De acuerdo), y 6 (Totalmente de acuerdo). El puntaje más bajo que se podía obtener en la versión original es 50 y el más alto es 300 , con lo cual a mayor puntuación obtenida en la escala, mayor discriminación percibe la persona.

\section{Procedimientos}

La recopilación de datos se llevó a cabo mediante el uso de cuestionarios a través de la plataforma PsychData utilizando como método de reclutamiento las redes sociales: Facebook, Twitter, Google+, WhatsApp, entre otras. Para proteger y 
garantizar los derechos de los participantes, utilizamos una hoja de consentimiento informado en la que se notificaba lo siguiente: (a) el propósito del estudio, (b) la naturaleza voluntaria del estudio, (c) los posibles riesgos y beneficios, (d) el derecho del participante a retirarse del estudio en cualquier momento, (e) la institución de procedencia y (f) los datos de identificación y contacto de los investigadores. Una vez recopilados los datos, analizamos los mismos utilizando el sistema para análisis estadísticos IBM SPSS versión 24.0 (IBM Corporation, 2016). Específicamente, se realizaron análisis descriptivos de la muestra, análisis factorial exploratorio, análisis de discriminación de ítems, análisis de confiabilidad, análisis de comparación y análisis de correlación entre los factores.

Para el análisis factorial exploratorio se utilizó el método de extracción de ejes principales con rotación oblicua para identificar las variables latentes que subyacen en los ítems. Se utilizó este procedimiento de ajuste por dos razones principales: (1) el método de extracción de ejes principales no se fundamenta en el supuesto de normalidad (Fabrigar, Wegener, MacCallum, \& Strahan, 1999) y (2) la rotación oblicua es más precisa y provee más información que la rotación ortogonal (Schmitt, 2011). Para determinar el número de factores se utilizaron dos criterios: el gráfico de sedimentación (Scree Test) y la cantidad de varianza explicada mediante el número de factores extraídos. El gráfico de sedimentación pretende identificar los factores cuyo valor asociado es lo suficientemente grande como para ser considerado. El punto de inflexión donde cambia la tendencia del gráfico, pasando de una pendiente pronunciada a una pendiente mínima, se toma como indicador del número de factores a extraer. En cuanto al segundo criterio, Hatcher (1994) sugiere que los factores a considerar sean aquellos que expliquen al menos un 5\% de la varianza. En este caso, dos factores cumplieron estos criterios. Como criterios de aceptación, se consideraron aquellos ítems con una carga factorial mayor a .50 en un solo factor (Stevens, 2002).

Para conocer la capacidad de discriminación de los ítems de los factores se calculó el índice de correlación ítem total $\left(r_{b i s}\right)$. Se identificaron como adecuados aquellos ítems cuyos valores estuviesen dentro de los puntos de corte .30 y .70 (Field, 2013). Para conocer el grado de confiabilidad de los factores, se calcularon el coeficiente alfa de Cronbach y división en mitades de Spearman-Brown. DeVellis (2017) indica que índices mayores a .70 son aceptables e índices entre .80 y .90 son buenos. Sin embargo, sugiere que en el caso de que los alfas sean mayores a .90 se revisen los ítems de la escala para considerar acortar la misma. Por su parte, Campo-Arias y Oviedo (2008) explican que índices superiores a .90 sugieren redundancia o duplicación de ítems, es decir, que por los menos un par de ítems miden exactamente el mismo aspecto del constructo y uno de ellos debe eliminarse.

Para el análisis factorial confirmatorio se utilizó el programa estadístico STATA versión 14.1 (StataCorp., 2017), con el método de estimación de máxima verosimilitud y las correcciones de Satorra y Bentler (2001). Para evaluar el ajuste del modelo se utilizaron los siguientes índices de bondad de ajuste: chi-cuadrado $\left(\chi^{2}\right)$, error medio cuadrático de aproximación (RMSEA), índice Tucker-Lewis (TLI) y el índice de ajuste comparativo (CFI). Para que exista un ajuste aceptable del modelo los valores de CFI y TLI deben ser $\geq .90$ y los valores del RMSEA deben ser $\leq .08$ (Byrne, 2010; Hu \& Bentler, 1999). Por su parte, los coeficientes de regresión de cada ítem en su respectivo factor debían superar el .50 (Hair, Black, Babin, Anderson, \& Tatham, 2006).

La correlación entre los factores del instrumento se calculó utilizando el coeficiente 


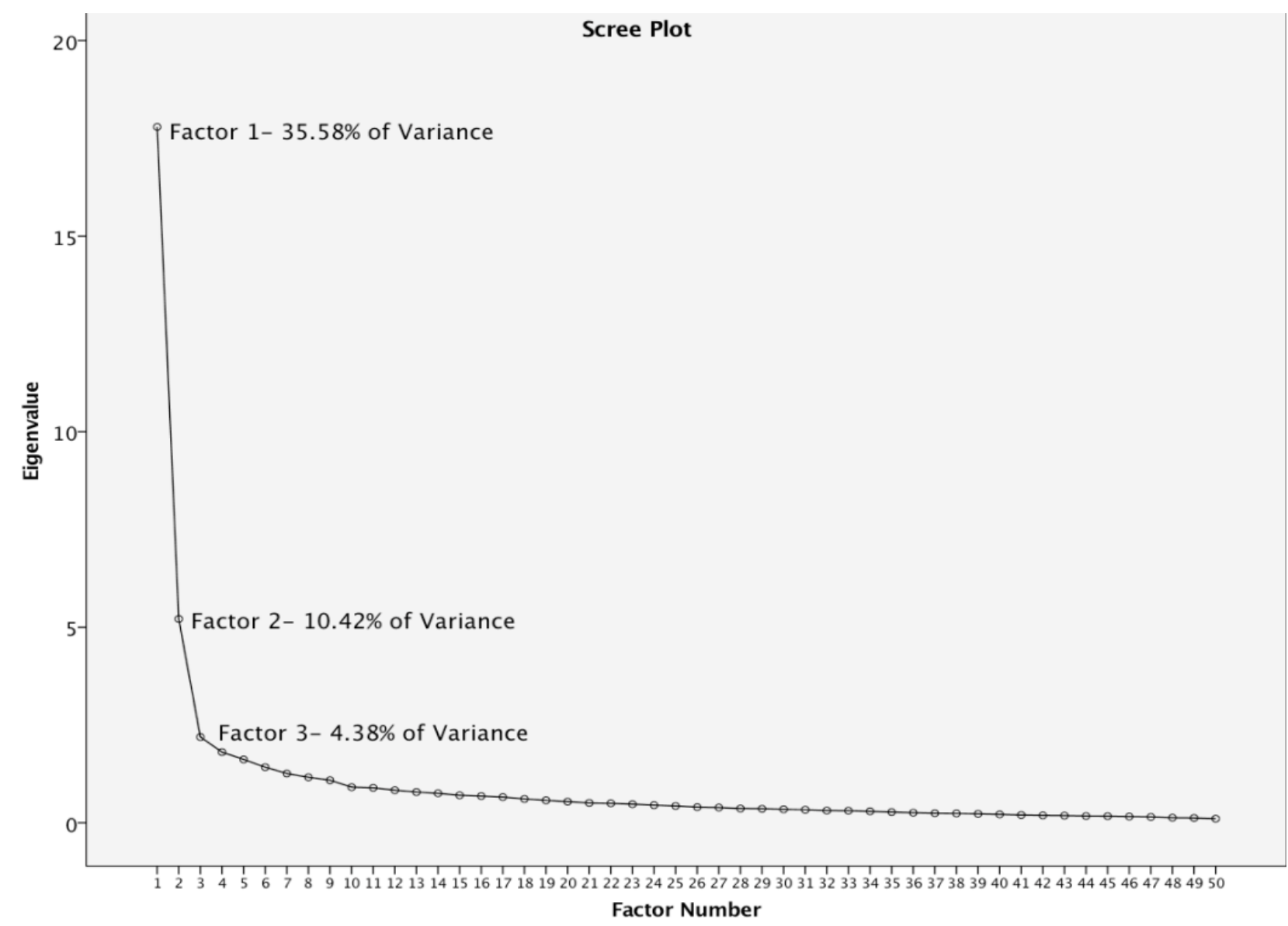

\section{Figura 1}

Gráfica de sedimentación y varianza explicada por los primeros factores.

producto-momento de Pearson $(r)$. Valores inferiores a .35 se consideran correlaciones débiles o bajas, valores entre .36 y .67 se consideran correlaciones moderadas, valores entre .68 y .89 se consideran correlaciones altas y, por último, valores de .90 en adelante se consideran correlaciones muy altas (Taylor, 1990). El tamaño del efecto de la correlación se calculó mediante el coeficiente de determinación $r^{2}$. Por su parte, las diferencias entre las medias de los factores se analizaron mediante la prueba $t$ y el tamaño del efecto con la $d$ de Cohen.

\section{Resultados}

Se realizó un primer análisis factorial exploratorio para evaluar la adecuación de los datos y auscultar cuantos factores explican 5\% o más de la varianza para ser retenidos. La Prueba de Kaiser-Meyer-Olkin apoyó la adecuación de los datos de muestreo para el análisis, $\mathrm{KMO}=.938$. La prueba de esfericidad de Bartlett fue significativa, $\chi_{(1225)}^{2}=10622.961, p<.001$, indicando que las correlaciones entre los reactivos fueron significativamente diferentes de cero, siendo este un indicador adicional de la adecuación para el análisis factorial. Considerando el criterio del 5\% de la varianza explicada y la gráfica de sedimentación, se decidió retener dos factores, ya que los factores 3 en adelante explicaban menos del $4.38 \%$ de la varianza (véase Figura 1). 
En los análisis factoriales posteriores, se consideraron como aceptables aquellos ítems con una carga factorial mayor a .50 en un solo factor. Al realizar el análisis restringido a dos factores, de los 50 ítems se eliminaron 10 ya que no cumplieron con los criterios de selección (ítems 17, 18, 20, 21, 25, 26, 27, 29, 37, 42). Luego, otros cuatro ítems fueron eliminados al no guardar relación teórica con los ítems identificados en su factor de mayor carga (ítems 38, 39, 40, 41). Este procedimiento redujo la escala a 36 ítems, 20 ítems en la subescala de discriminación individual y 16 ítems para la subescala de discriminación grupal. En la Tabla 2 se pueden apreciar las cargas factoriales de esta versión de la escala.

Los ítems correspondientes a cada factor fueron sometidos a análisis de discriminación mediante el cálculo del índice de correlación ítem total $\left(r_{b i s}\right)$ y a análisis de confiabilidad. Al observar los valores obtenidos en el total de correlación por ítem se identificaron y eliminaron ocho ítems $(5,7,10,11,14,24,33$ y 35) con índices fuera de los puntos de corte $\left(r_{b i s}>.30 \mathrm{y}<.70\right)$. Además, dado que las subescalas obtuvieron un coeficiente alfa de Cronbach de .92, se examinaron los ítems para identificar redundancia o duplicidad en el contenido de los mismos. Se observó que los ítems 4, 9, 19, 23, 30, 32, 34 y 36 miden aspectos semejantes a otros ítems de sus correspondientes subescalas, por lo cual fueron eliminados. Esto redujo la escala a 20 ítems, 10 ítems en la subescala de discriminación individual y 10 ítems para la subescala de discriminación grupal. Estos 20 ítems de la última versión fueron sometidos a análisis factorial exploratorio y a análisis de discriminación mediante el cálculo del índice de correlación ítem-total.

El análisis factorial exploratorio mostró nuevamente una estructura de dos factores que explicaban el 52\% de la varianza, del cual el 39\% lo explica el factor 1 y el $13 \%$ lo explica el factor 2 .
Al revisar las cargas factoriales de estos reactivos, se observó que todos cumplieran con los criterios de inclusión. Las pruebas de Kaiser-Meyer-Olkin $(\mathrm{KMO}=.907)$ y la prueba de esfericidad Bartlett $\left(\chi_{(190)}^{2}=3004.659, p<.001\right)$ apoyaron la adecuación de los datos de muestreo para el análisis. A su vez, el análisis de discriminación de los ítems reveló que todos los índices están dentro de los puntos de corte sugeridos por Field (2013). En la Tabla 3 se presentan las cargas factoriales y los índices de discriminación obtenidos por los ítems en su respectiva subescala.

Los 20 ítems de la escala final fueron sometidos a un análisis factorial confirmatorio con ecuaciones estructurales. El modelo puesto a prueba estaba conformado por dos factores latentes: discriminación individual y discriminación grupal (véase Figura 1). Los resultados arrojaron un ajuste adecuado para el modelo con las correspondientes liberaciones entre errores, $\chi_{(190)}^{2}=388.41$, $p<.001$; $\mathrm{RMSEA}=.07$; CFI $=.92$; TLI $=.91$; $\chi_{\text {corregido(190) }}^{2}=320.82, p<.001 ; \mathrm{RMSEA}_{\text {corregido }}=$ $.06 ; \mathrm{CFI}_{\text {corregido }}=.93 ; \mathrm{TLI}_{\text {corregido }}=.92$. A su vez, se examinaron los coeficientes de regresión de cada ítem, esperando que cada uno de estos fuese mayor a .50. Los coeficientes de regresión fluctuaron entre .56 y .75 (véase Tabla 3).

Luego, se analizó la confiabilidad de las dos subescalas de la versión final del instrumento. Para ello se utilizaron dos métodos: el coeficiente alfa de Cronbach y división en mitades de Spearman-Brown. Ambas subescalas mostraron coeficientes alfa de Cronbach de .89 y coeficientes Spearman-Brown de .86. Por último, se analizó la relación entre las subescalas mediante la correlación $r$ de Pearson $\left(r=.51, p<.001, r^{2}\right.$ $=.26$ ) y las diferencias entre las medias de las subescalas mediante la prueba $t$. El análisis de comparación reflejó que los participantes perciben estadísticamente más discriminación grupal $(\mathrm{M}=50.45)$ que discriminación individual 
Tabla 2

Cargas factoriales superiores a .50 en la Versión de 36 ítems de la escala.

\begin{tabular}{llc}
\hline Ítems & 1 & 2 \\
\hline
\end{tabular}

14. He recibido insultos de personas extrañas por ser LGBT.

.79

5. He sido menospreciado públicamente por mi orientación sexual. $\quad .78$

24. He sido discriminado por manifestar abiertamente mi orientación sexual. $\quad .78$

10. He recibido insultos por mi orientación sexual. $\quad .76$

7. He sido acosado verbalmente por mi orientación sexual. $\quad .76$

11. Se han burlado de mí por ser LGBT. $\quad .72$

13. Me han puesto sobrenombres despectivos por mi orientación sexual. $\quad .71$

15. He recibido insultos de personas conocidas por ser LGBT. $\quad .71$

6. He sido juzgado por no ser heterosexual. $\quad .71$

4. He experimentado personalmente prejuicio y discrimen por mi orientación sexual. $\quad .70$

$\begin{array}{ll}\text { 8. He sido acosado físicamente por ser LGBT. } & .68\end{array}$

22. He sido víctima de bullying por mi orientación sexual. $\quad .67$

2. Me tratan diferente porque no soy heterosexual. $\quad .67$

3. En ciertos ambientes siento que las personas me rechazan porque soy LGBT. 63

23. He sido víctima de bullying a través de las redes sociales por mi orientación sexual. $\quad 62$

12. He sido tratado injustamente en mi trabajo o lugar de estudios por ser LGBT.

9. He sido agredido físicamente por ser LGBT.

1. La gente generalmente piensa que soy inmoral porque soy LGBT. 58

19. Mis compañeros de trabajo o estudios me han rechazado por ser LGBT.

16. He sido tratado injustamente en restaurantes o tiendas por mi orientación sexual. 57

48. El sistema educativo del país no se ajusta a las necesidades de la comunidad LGBT. .73

36. La legislatura de mi país discrimina a la comunidad LGBT.

35. Las personas piensan que la orientación sexual de la comunidad LGBT es abominable. 72

46. Las personas LGBT son generalmente excluidas de grupos religiosos/iglesias. .70

50. La comunidad LGBT carece de servicios especializados.

33. En mi país, asocian a la comunidad LGBT con enfermedades de transmisión sexual. 69

34. En las escuelas de mi país no se enseña sobre las contribuciones importantes de... .67

43. En público, las parejas del mismo sexo no son tratadas de la misma forma que las... .67

31. Los políticos de mi país minimizan las necesidades de la comunidad LGBT. 66

32. En mi país, se piensa que todas las personas LGBT son promiscuas.

47. La sociedad no apoyaría a un candidato LGBT que aspire a un puesto político. .64

44. Las personas suelen tratar irrespetuosamente a la comunidad LGBT. .63

45. Generalmente, las personas LGBT no son aceptadas socialmente. 57

49. En donde vivo, las personas LGBT son vistas como pecadores.

30. Las leyes de mi país marginan a la comunidad LGBT.

28. En mi país, la comunidad LGBT es objeto de burlas.

Nota. Factor 1 = Discriminación individual; Factor $2=$ Discriminación grupal. KMO = 939 . 


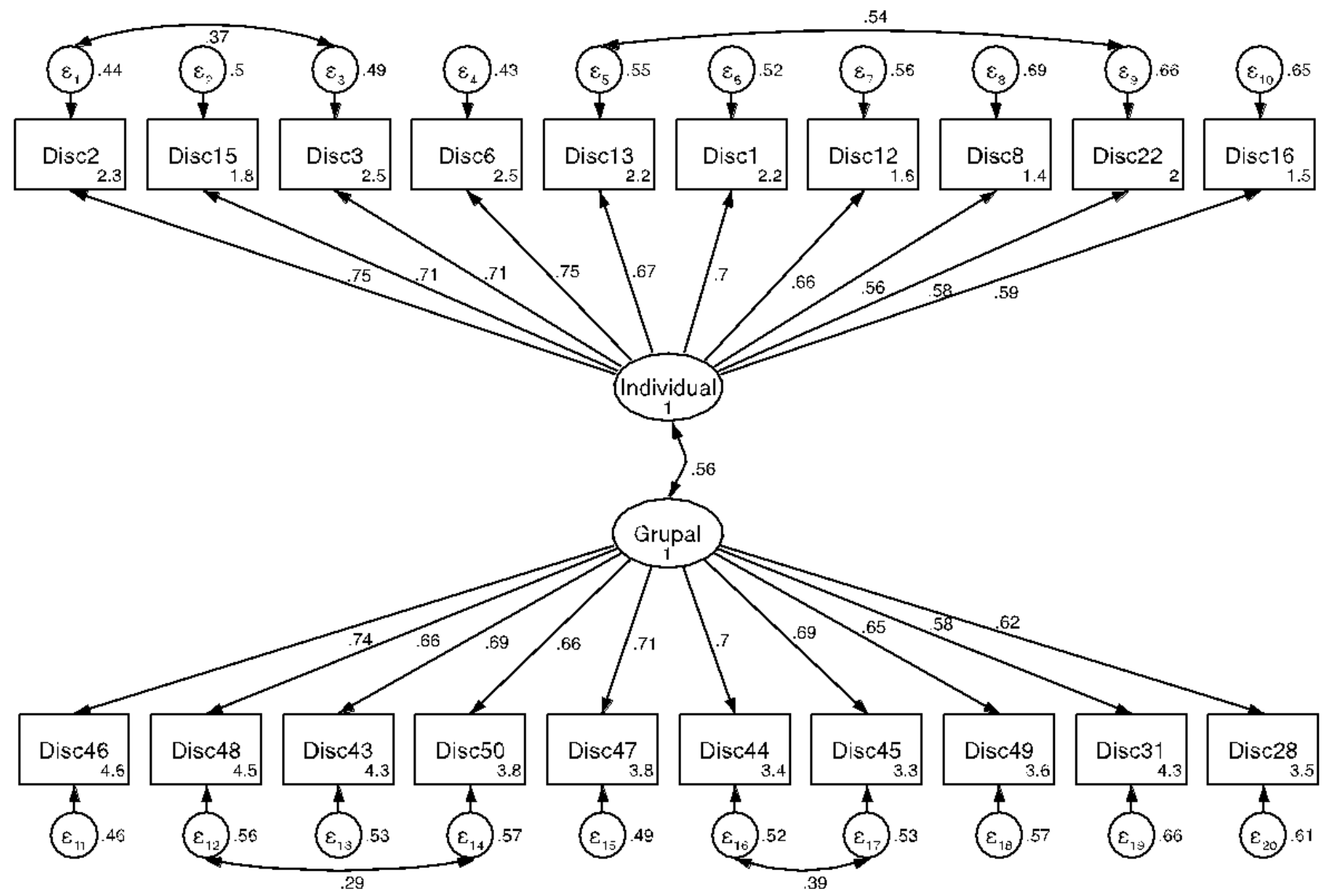

Figura 1

Modelo final de la Escala de Discriminación Percibida en la Comunidad LGBT.

$(\mathrm{M}=35.13), t_{(304)}=-23.600, \mathrm{p}<.001, d=1.38$. La Tabla 4 muestra los estadísticos descriptivos de las subescalas, los coeficientes de confiabilidad y las correlaciones entres las subescalas del instrumento.

\section{Discusión}

En Puerto Rico, así como en muchos países del mundo, la comunidad LGBT es víctima de discrimen, prejuicio y rechazo social. Sin embargo, la comunidad científica del país no cuenta con un instrumento diseñado para medir la discriminación percibida por los miembros de la comuni- dad LGBT en escenarios clínicos, sociales o académicos. Es por ello que el presente estudio tuvo como propósito desarrollar, validar y examinar las propiedades psicométricas de la Escala de Discriminación Percibida en la Comunidad LGBT. Los resultados confirman que el instrumento posee las propiedades psicométricas adecuadas para medir discriminación percibida desde dos dimensiones claramente distinguibles: discriminación individual y discriminación grupal. A su vez, los índices de confiabilidad alcanzados respaldan empíricamente la consistencia interna de las dos subescalas del instrumento.

En términos teóricos, el análisis factorial exploratorio reveló la existencia de dos factores 
Tabla 3

Cargas factoriales, índices de discriminación y coeficientes de regresión de los ítems.

\begin{tabular}{|c|c|c|c|c|}
\hline Ítem & 1 & 2 & $r_{b i s}$ & $\beta$ \\
\hline 2. Me tratan diferente porque no soy heterosexual. & .76 & & .69 & .75 \\
\hline 15. He recibido insultos de personas conocidas por ser LGBT. & .71 & & .67 & .71 \\
\hline 3. En ciertos ambientes siento que las personas me rechazan porque soy LGBT. & .71 & & .66 & .71 \\
\hline 6. He sido juzgado por no ser heterosexual. & .70 & & .69 & .75 \\
\hline 13. Me han puesto sobrenombres despectivos por mi orientación sexual. & .68 & & .68 & .67 \\
\hline 1. La gente generalmente piensa que soy inmoral porque soy LGBT. & .67 & & .63 & .70 \\
\hline 12. He sido tratado injustamente en mi trabajo o lugar de estudios por ser... & .66 & & .62 & .66 \\
\hline 8. He sido acosado físicamente por ser LGBT. & .61 & & .55 & .56 \\
\hline 22. He sido víctima de bullying por mi orientación sexual. & .61 & & .60 & .58 \\
\hline 16. He sido tratado injustamente en restaurantes o tiendas por mi orientación... & .59 & & .55 & .59 \\
\hline 46. Las personas LGBT son generalmente excluidas de grupos religiosos... & & .75 & .69 & .74 \\
\hline 48. El sistema educativo del país no se ajusta a las necesidades de la... & & .75 & .64 & .66 \\
\hline 43. En público, las parejas del mismo sexo no son tratadas de la misma... & & .74 & .65 & .69 \\
\hline 50. La comunidad LGBT carece de servicios especializados. & & .72 & .64 & .66 \\
\hline 47. La sociedad no apoyaría a un candidato LGBT que aspire a un puesto... & & .72 & .66 & .71 \\
\hline 44. Las personas suelen tratar irrespetuosamente a la comunidad LGBT. & & .64 & .68 & .70 \\
\hline 45. Generalmente, las personas LGBT no son aceptadas socialmente. & & .64 & .66 & .69 \\
\hline 49. En donde vivo, las personas LGBT son vistas como pecadores. & & .58 & .61 & .65 \\
\hline 31. Los políticos de mi país minimizan las necesidades de la comunidad LGBT. & & .53 & .54 & .58 \\
\hline 28. En mi país, la comunidad LGBT es objeto de burlas. & & .51 & .57 & .62 \\
\hline
\end{tabular}

Nota. Factor 1 = Discriminación individual (análisis factorial exploratorio); Factor 2 = Discriminación grupal (análisis factorial exploratorio); $r_{b i s}=$ índice de discriminación del ítem en su respectivo factor; $\beta$ = coeficientes de regresión del ítem en su respectivo factor (análisis factorial confirmatorio).

Tabla 4

Medias, desviaciones estándar, alfas y correlaciones entres las subescalas.

\begin{tabular}{lcccccc}
\hline \multicolumn{1}{c}{ Factor } & $\mathbf{M}$ & DE & $\alpha$ & $\begin{array}{c}\text { Spear- } \\
\text { man-Brown }\end{array}$ & $\mathbf{1}$ & $\mathbf{2}$ \\
\hline Discriminación Individual & 35.13 & 12.69 & .89 & .86 & - & .56 \\
Discriminación Grupal & 50.45 & 9.31 & .89 & .86 & .51 & - \\
\hline
\end{tabular}

Nota. $\mathrm{M}=$ media; $\mathrm{DE}=$ desviación estándar; $\alpha$ = alfa de Cronbach. La correlación sobre la diagonal representa la correlación entre los factores latentes, mientras que la correlación bajo la diagonal representa la correlación de las puntuaciones directas. Todas las correlaciones fueron significativas a $p<.001$. $(\mathrm{N}=305)$. 
latentes en los ítems del instrumento, que fueron certificados posteriormente por el análisis factorial confirmatorio. Este hallazgo reproduce la lógica teórica utilizada por los autores en la redacción de los ítems del instrumento: discriminación individual y discriminación grupal. Estos dos factores deben considerarse escalas independientes que examinan dimensiones distintas de la discriminación percibida. De hecho, la diferencia estadísticamente significativa entre las medias de las subescalas y la correlación moderada entre los factores sugieren dos variables claramente diferenciables. La primera subescala, discriminación individual, evalúa las experiencias personales de discrimen percibidas por el individuo. Es decir, mide conductas discriminatorias concretas que el individuo experimenta en su contra como, por ejemplo, trato diferente, insultos, rechazo, prejuicio, injusticias, acoso físico y bullying. La literatura científica ha asociado consistentemente estas experiencias personales con deterioros significativos en la salud mental de la comunidad LGBT (Burges et al., 2007; Sandfort et al., 2003; Singh \& Dickey, 2016; Woodford et al., 2015).

Por su parte, la segunda subescala, discriminación grupal, evalúa la percepción que tiene el individuo sobre las acciones y conductas discriminatorias dirigidas al colectivo LGBT en su conjunto, tales como exclusión religiosa, poca visibilidad en la política pública, desigualdad educativa, carencia de servicios especializados, rechazo social generalizado, sobregeneralizaciones morales y burla colectiva. Como otra importante aportación teórica respecto a otros estudios, nuestros resultados confirmaron lo que investigaciones previas han encontrado en otros grupos minoritarios (Lahoz-Ubach \& Forns-Santacana, 2016), que los miembros de la comunidad LGBT perciben un mayor nivel de discriminación dirigida al grupo como un colectivo, en comparación con la discriminación que perciben hacia su per- sona en particular. Este dato puede interpretarse como una disidencia poco cré́ble, aunque cobra sentido cuando se entiende como una protección del yo frente a la dolorosa aceptación de las desigualdades sociales (Moreno-Salas, 2011). Estos hallazgos robustecen la teoría bifactorial de discriminación percibida que sustenta los supuestos teóricos del instrumento.

En cuanto a la confiabilidad de la escala, se obtuvieron índices superiores al mínimo recomendado por la literatura científica en ambas subescalas (DeVellis, 2017), lo cual sugiere que la versión final de la escala es un instrumento estable, reproducible y consistente para la medición de discriminación percibida en la comunidad LGBT. Asimismo, las correlaciones de cada ítem con la puntuación total manifiestan una consistencia interna notable. Esto sugiere que los ítems de la versión final discriminan adecuadamente y son capaces de diferenciar personas con distintos niveles de discriminación percibida.

En términos prácticos, se demostró que la versión final de la Escala de Discriminación Percibida en la Comunidad LGBT puede ser utilizada para el desarrollo de nuevas investigaciones en el campo de la psicología caribeña. Este es un gran avance dado que, en Puerto Rico y el Caribe, no existía un instrumento que examinara este fenómeno. Además, puede facilitar a los psicoterapeutas la realización de cernimientos y avalúos para entender cómo la discriminación percibida por el individuo LGBT afecta perniciosamente su salud mental y su bienestar integral. Investigaciones que datan de la década de 1950 han demostrado que la percepción de discriminación y la internalización del prejuicio impactan directamente en el bienestar psicológico, la insatisfacción personal, el rechazo a sí mismo y exacerban los niveles de estrés, ansiedad y depresión (Allport, 1954; Banks, Kohn-Wood, \& Spencer, 2006; LahozUbach \& Forns-Santacana, 2016; Lewin, 1952). 
Por esta razón, junto con la evidencia empírica de las experiencias discriminatorias hacia la comunidad LGBT de Puerto Rico (González-Guardarrama \& Toro-Alfonso, 2012), este instrumento se constituye como una herramienta práctica $\mathrm{y}$ efectiva en el quehacer investigativo de los profesionales de la conducta.

La versión final del instrumento consta de 20 ítems distribuidos en dos subescalas (10 ítems en cada una) fundamentadas en el marco teórico bifactorial de discriminación percibida (individual y grupal) desarrollado en otras investigaciones (Armenta \& Hunt, 2009; González-Rivera et al., 2017). Las puntuaciones deben ser calculadas mediante la sumatoria de los 10 ítems de cada subescala por separado para obtener una puntuación específica por tipo de discriminación percibida, individual o grupal. Considerando la independencia de los constructos y las diferencias significativas entre las medias de las subescalas, no debe generarse una medida con la sumatoria de ambas subescalas. El orden de los ítems en la versión final fue determinado por categorías: los primeros 10 ítems corresponden a la subescala de discriminación individual, y los últimos 10, a subescala de discriminación grupal. Las puntuaciones posibles en ambas subescalas fluctúan de 10 a 60 . Se entiende que, a mayor puntuación obtenida en cada subescala, mayor discriminación percibe la persona.

\section{Limitaciones y Fortalezas}

Al igual que toda investigación, nuestro estudio no está exento de limitaciones. Primero, la muestra fue recopilada de forma accidental y no fue aleatoria; sin embargo, fue amplia y heterogénea. Segundo, no se pudo establecer la confiabilidad del instrumento a través del tiempo, solo se pudo hacer a través de sus elementos. No obstan- te, las avanzadas técnicas utilizadas en el estudio brindan robustez empírica a nuestros resultados. Tercero, el procedimiento para recoger los datos no fue estandarizado, esto puede afectar las medias del estudio y aumentar el error estándar de medición. Por último, los dos análisis factoriales fueron realizados con la misma muestra, lo cual no es aconsejado.

A pesar de sus limitaciones, la presente investigación tiene varias fortalezas que vale la pena enumerar. En primer lugar, es el primer instrumento desarrollado y validado en Puerto Rico para medir discriminación percibida en la comunidad LGBT. Segundo, nuestro estudio robustece la teoría bifactorial de discriminación percibida, lo que provee evidencia teórica y empírica sobre la estructura factorial del instrumento. Por último, ofrece a la comunidad científica hispanoparlante un instrumento confiable y válido que enriquecerá las investigaciones dirigidas a comprender las formas en que los miembros de la comunidad LGBT perciben el prejuicio, el rechazo y la exclusión.

\section{Futuras Investigaciones}

Para futuras investigaciones se recomienda aplicar la escala a otra muestra de participantes para realizar el proceso de validación cruzada. También sería un valor añadido examinar la confiabilidad temporal a través de la técnica de prueba-reprueba y realizar un nuevo análisis factorial confirmatorio. Se recomienda que la Escala de Discriminación Percibida en la Comunidad LGBT se valide en otras poblaciones latinoamericanas para auscultar sus propiedades psicométricas en distintos contextos nacionales e internacionales. Esto permitirá comparar el comportamiento de la escala en distintos contextos internacionales y facilitará estudiar el fenómeno de la discriminación hacia esta comunidad desde una perspectiva 
multicultural.

En conclusión, el presente estudio demostró que la Escala de Discriminación Percibida en la Comunidad LGBT cuenta con propiedades psicométricas apropiadas, lo que implica una alta confiabilidad y una sólida estructura interna de dos factores latentes. Ante esto, se concluye que el instrumento es útil para investigar el fenómeno de la discriminación percibido en la comunidad LGBT. Se espera que el instrumento desarrollado sea de beneficio para su uso en ámbitos de aplicación y de investigación.

\section{Referencias}

Allport, G. W. (1954). The nature of prejudice. Cambridge, MA: Addison-Wesley.

Armenta, B. E., \& Hunt, J. S. (2009). Responding to societal devaluation: Effects of perceived personal and group discrimination on the group identification and self-esteem of Latinos/as. Group Processes \& Intergroup Relations, 12(1), 23-39. doi: 10.1177/1368430208098775

Ato, M., López, J. J., \& Benavente, A. (2013). Un sistema de clasificación de los diseños de investigación en psicología. Anales de Psicología, 29(3), 1038-1059. Recuperado de http://revistas.um.es/analesps

Banks, K. H., Kohn-Wood, L. P., \& Spencer, M. (2006). An examination of the African American experience of everyday discrimination and symptoms of psychological distress. Community Mental Health Journal, 42(6), 555-570. doi: 10.1007/s10597-006-9052-9

Bostwick, W. B., Boyd, C. J., Hughes, T. L., West, B. T., \& McCabe, S. E. (2014). Discrimination and mental health among lesbian, gay, and bisexual adults in the United States. American Journal of Orthopsychiatry, 84(1), 35-45. doi: 10.1037/h0098851

Burges, D., Lee, R., Tran, A., \& Van Ryn, M. (2007). Effects of perceived discrimination on mental health and mental health services utilization among gay, lesbian, bisexual and transgender persons. Journal of LGBT Health Research, 3(4), 1-14. doi: 10.1080/15574090802226626

Byrne, B. M. (2010). Structural equation modeling with AMOS: Basic concepts, applications, and programming ( $2^{\mathrm{a}}$ ed.). New York, NY: Taylor \& Francis Group.

Campo-Arias, A., \& Oviedo, H. C. (2008). Propiedades psicométricas de una escala: La consistencia interna. Revista de Salud Pública, 10(5), 831-839. doi: 10.1590/s0124-00642008000500015

DeVellis, R. F. (2017). Scale development: Theory and applications ( $\left.4^{\mathrm{a}} \mathrm{Ed}\right)$. California: Sage Publications.

Fabrigar, L. R., Wegener, D. T., MacCallum, R. C., \& Strahan, E. J. (1999). Evaluating the use of exploratory factor analysis in psychological research. Psychological Methods, 4(3), 272-299. doi: 10.1037/1082-989x.4.3.272

Fernández-Rodríguez, M. del C., \& Calderón-Squiabro, J. (2014). Prejuicio y distancia social hacia personas homosexuales por parte de jóvenes universitarios. Revista Puertorriqueña de Psicología, 25(1), 52-60. Recuperado de http://www.ojs.repsasppr.net/index. php/reps/index

Field, A. P. (2013). Discovering statistics using SPSS. London, England: SAGE.

Fingerhut, A. W., Peplau, L. A., \& Gable, S. L. (2010). Identity, minority stress and psychological well-being among gay men and lesbians. Psychology \& Sexuality, 1(2), 101-114. doi: 10.1080/19419899.2010.484592

Francia-Martínez, M., Esteban, C., \& Lespier, Z. (2017). Actitudes, conocimiento y distancia social de psicoterapeutas con la comunidad transgénero y transexual. Revista Puertorriqueña de Psicología, 28(1), 98-113. Recuperado de http://www.ojs.repsasppr.net/ index.php/reps/index

González-Guardarrama, J., \& Toro-Alfonso, J. (2012). El significado de la experiencia de la aceptación de la orientación sexual homosexual desde la memoria de un grupo de hombres adultos puertorriqueños. Eureka, 9(2), 158-170. Recuperado de 
http://psicoeureka.com.py

González-Rivera, J. A., Pabellón-Lebrón, S., \& Rosario-Rodríguez, A. (2017). El rol mediador de la identificación ateísta en la relación entre discriminación y bienestar psicológico: Un estudio preliminar. Revista Puertorriqueña de Psicología, 28(2), 406-421. Recuperado de http://www.ojs.repsasppr.net/index.php/ reps/index

Hair, J. F., Black, W. C., Babin, B. J., Anderson, R. E., \& Tatham, R. L. (2006). Multivariate Data Analysis (6 ed.). New Jersey, NJ: Prentice-Hall International.

Hatcher, L. (1994). A step-by-step approach to using the SAS system for factor analysis and structural equation modeling. Cary, NC: SAS Institute.

Hu, L. T., \& Bentler, P. M. (1999). Cutoff criteria for fit indexes in covariance structure analysis: Conventional criteria versus new alternatives. Structural Equation Modeling: A Multidisciplinary Journal, 6(1), 1-55. doi: 10.1080/10705519909540118

IBM Corporation (2016). IBM SPSS Statistics for Windows, Version 24.0 [Software de computación]. Armonk, NY: IBM.

Lahoz-Ubach, S., \& Forns-Santacana, M. (2016). Discriminación percibida, afrontamiento y salud mental en migrantes peruanos en Santiago de Chile. Psicoperspectivas, 15(1), 157-168. doi: 10.5027/ psicoperspectivas-vol15-issue1-fulltext-613

Lewin, K. (1952). Field theory in social science. New York, NY: Haper and Row.

Luiggi-Hernández, J. G., Laborde-Torres, G. E., González-Domínguez, J., Carrasquillo-Sánchez, G. M, Piñero-Meléndez, M., Castro-Medina, D. M., \& González-Rentas, C. D. (2015). Outing the discrimination towards LGBT people during the hiring process: What about their well-being? Revista Puertorriqueña de Psicología, 26(2), 194-213. Recuperado de http://www.ojs.repsasppr.net/index.php/reps/ index

Marchueta-Perez, A. (2014). Consecuencias del bullying homofóbico retrospectivo y los factores psicosociales en el bienestar psicológico de sujetos LGBT.
Revista de Investigación Educativa, 32(1), 255-271. doi: 10.6018/rie.32.1.168461

Montero, I., \& León, O. G. (2007). A guide for naming research studies in psychology. International Journal of Clinical and Health Psychology, 7(3), 847-862. Recuperado de http://www.aepc.es/ijchp

Moreno-Salas, M. (2011). Percepción de discriminación social en un grupo indígena costarricense: Los huetares de Quitirrisí. Actualidades en Psicología, 25(112), 117-134. Recuperado de https://revistas.ucr.ac.cr/index.php/actualidades

Nazario-Serrano, J. (2016). Desde la demonización a la aceptación: La religión y la espiritualidad como factores protectores o factores de riesgo en la población LGBT. En M. Vázquez-Rivera, A. Martínez-Taboas, M. Francia-Martínez y J. Toro-Alfonso (Eds.), LGBT 101: Una mirada introductoria al colectivo (pp. 247-266). Hato Rey, Puerto Rico: Publicaciones Puertorriqueñas.

Nieves-Rosa, L. E. (2012). Homofobia al estilo universitario. Revista Puertorriqueña de Psicología, 23(2), 62-76. Recuperado de http://www.ojs.repsasppr.net/ index.php/reps/index

Rivera-Quiñones, C., Toro-Alfonso, J., \& Meléndez, L. (2013). Minorías frente al alza en la criminalidad: Percepción de seguridad de la comunidad lesbiana, gay, bisexual y transgénero (LGBT) en Puerto Rico. Revista Puertorriqueña de Psicología, 24(2), 1-14. Recuperado de http://www.ojs.repsasppr.net/index. $\mathrm{php} / \mathrm{reps} / \mathrm{index}$

Rodríguez-Díaz, C. E., Jovet-Toledo, G. G., Vélez-Vega, C. M., Ortiz-Sánchez, E. J., Santiago-Rodríguez, E. I, Vargas-Molina, R. L. ... Mulinelli-Rodríguez, J. J. (2016). Discrimination and health among lesbian, gay, bisexual and trans people in Puerto Rico. Puerto Rico Health Science Journal, 35(3), 154-159. Recuperado de http://prhsj.rcm.upr.edu/index.html

Rodríguez-Díaz, C. E., Martínez-Vélez, J. J., Jovet-Toledo, G. G., Vélez-Vega, C. M., Hernández-Otero, N., Escotto-Morales, B., \& Mulinelli-Rodríguez, J. J. (2016). Challenges for the well-being of and health 
equity for lesbian, gay, and bisexual people in Puerto Rico. International Journal of Sexual Health, 28(4), 286-295. doi: 10.1080/19317611.2016.1223252

Rodríguez-Madera, S., Ramos-Pibernus, A., Padilla, M., \& Varas-Díaz, N. (2016). Radiografías de las comunidades trans en Puerto Rico: Visibilizando feminidades y masculinidades alternas. En M. Vázquez-Rivera, A. Martínez-Taboas, M. Francia-Martínez y J. Toro-Alfonso (Eds.), LGBT 101: Una mirada introductoria al colectivo (pp. 315-342). Hato Rey, Puerto Rico: Publicaciones Puertorriqueñas.

Rosario-Hernández, E., Rovira-Millán, L. V., Luna-Cruz, C. F., Neris, M., \& Acevedo, G. (2009). Saliendo del clóset en el trabajo: La relación entre el manejo de la identidad sexual, heterosexismo organizacional percibido, actitudes de trabajo y bienestar psicológico. Revista Puertorriqueña de Psicología, 20(1), 103143. Recuperado de http://www.ojs.repsasppr.net/ index.php/reps/index

Sandfort, T. G. M., De Graaf, R., \& Bijl, R. V. (2003). Same-sex sexuality and quality of life: Findings from the Netherlands Mental Health Survey and incidence study. Archives of Sexual Behavior, 32(1), 15-22. doi: 10.1023/A:1021885127560

Satorra, A., \& Bentler, P. M. (2001). A scaled difference chi-square test statistic for moment structure analysis. Psychometrika, 66(4), 507-514. doi: 10.1007/ BF02296192

Schmitt, T. A. (2011). Current methodological considerations in exploratory and confirmatory factor analysis. Journal of Psychoeducational Assessment, 29(4), 304-321. doi: 10.1177/0734282911406653

Singh, A. A., \& Dickey, L. M. (2016). Implementing the APA guidelines on psychological practice with transgender and gender nonconforming people: A call to action to the field of psychology. Psychology of Sexual Orientation and Gender Diversity, 3(2), 195-200. doi: $10.1037 /$ sgd0000179

StataCorp. (2017). Stata: Release 15 [Software de computación]. College Station, TX: StataCorp LLC.

Stevens, J. (2002). Applied multivariate statistics for the social sciences (4 ${ }^{\mathrm{a}}$ ed.). Mahwah, NJ: Lawrence Erlbaum Associates.

Taylor, R. (1990). Interpretation of the correlation coefficient: A basic review. Journal of Diagnostic Medical Sonography, 6(1), 35-39. doi: 10.1177/875647939000600106

Woodford, M. R., Paceley, M. S., Kulick, A., \& Hung, J. S. (2015). The LGBT social climate matters: Policies, protests, and placards and psychological well-being among LGBT emerging adults. Journal of Gay \& Lesbian Social Services, 27(1), 116-141. doi: 10.1080/10538720.2015.990334 